\title{
Spectrum of movement disorders in GNAO1 encephalopathy: in-depth phenotyping and case-by-case analysis
}

Soo Yeon Kim 1,2, YoungKyu Shim', Young Joon Ko' , Soojin Park ${ }^{1,3}$, Se Song Jang ${ }^{1}$, Byung Chan Lim 1,2,4, Ki Joong Kim ${ }^{1,4}$ and Jong-Hee Chae $1,2,4^{*}$ (D)

\begin{abstract}
Background: GNAO1 encephalopathy is a rare neurodevelopmental disorder characterized by distinct movement presentations and early onset epileptic encephalopathy. Here, we report the in-depth phenotyping of genetically confirmed patients with GNAO1 encephalopathy, focusing on movement presentations.

Results: Six patients who participated in Korean Undiagnosed Disease Program were diagnosed to have pathogenic or likely pathogenic variants in GNAO1 using whole exome sequencing. All medical records and personal video clips were analyzed with a literature review. Three of the 6 patients were male. Median follow-up duration was 41 months (range 7-78 months) and age at last examination was 7.4 years (range 3.3-16.9 years). Initial complaints were hypotonia or developmental delay in 5 and right-hand clumsiness in 1 patient, which were noticed at median age of 3 months (range 0-75 months). All patients showed global developmental delay and 4 had severely retarded development. Five patients $(5 / 6,83.3 \%)$ had many different movement symptoms with various onset and progression. The symptoms included stereotyped hands movement, non-epileptic myoclonus, dyskinesia, dystonia and choreoathetosis. Whole exome sequencing identified 6 different variants in GNAO1. Three were novel de novo variants and atypical presentation was noted in a patient. One variant turned out to be inherited from patient's mother who had mosaic variant. Distinct and characteristics movement phenotypes in patients with variant p.Glu246Lys and p.Arg209His were elucidated by in-depth phenotyping and literature review.
\end{abstract}

Conclusions: We reported 6 patients with GNAO1 encephalopathy showing an extremely diverse clinical spectrum on video. Some characteristic movement features identified by careful inspection may also provide important diagnostic insight and practice guidelines.

Keywords: GNAO1, GNAO1 encephalopathy, Movement disorder, Early-onset dystonia, Early-onset chorea

\section{Introduction}

Since the first identification of GNAO1 as a new causative gene of early-onset epileptic encephalopathy in 2013, variable phenotypes have been reported [1-3]. Epilepsy

\footnotetext{
*Correspondence: chaeped1@snu.ac.kr

${ }^{1}$ Division of Pediatric Neurology, Department of Pediatrics, Pediatric

Clinical Neuroscience Center, Seoul National University Children's Hospital, Seoul, Korea

Full list of author information is available at the end of the article
}

itself varied from early onset epileptic encephalopathy including Ohtahara syndrome, and generalized and focal epilepsies of different ages [1, 4, 5]. Movement phenotypes were also reported since the first clinical report and have recently become major presenting symptoms: so far, chorea, dystonia, orofacial dyskinesia, and stereotyped hand movements have been reported in patients of different ages, associated with GNAO1 [1, 6-8]. Developmental milestones are also delayed in most patients with GNAO1 variants and the degree of developmental 
delay varies from neonatal hypotonia to intellectual disability [7-9]. Interestingly, most patients mainly present phenotypes between the epilepsy or movement disorder, whereas a small number of patients showed both epilepsy and movement phenotypes equally [10]. Many studies suggested that different locations or different functional changes of variants lead to separate phenotypes [1, 10-12]. Gain-of-function mutation (GOM) turned out to be associated with movement disorder [11]. However, it could not explain all the cases. Further studies on phenotype, genotype, and molecular pathways are certainly required for better understanding. Phenotypes often mimic other neurodevelopmental disorders and are barely immediately recognizable because the disease usually occurs in infancy with nonspecific symptoms and evolves over time. Therefore, comprehensive and serial phenotyping would be the very first step to grasp the disease and establish further functional studies.

Here, we report 6 pediatric patients who carried GNAO1 variants identified from the Korean Undiagnosed Disease Program (KUDP), with the aim of delineating detailed phenotypes and characterizing their phenotype-genotype association with a comprehensive review of previously reported cases.

\section{Patients and methods}

\section{Patients and study approval}

Six patients who carried GNAO1 variants were enrolled in this study. All patients were diagnosed using whole exome sequencing (WES) after participating in the KUDP, which launched in 2017 [13]. The entire KUDP protocol including diagnostic process and data sharing was approved by the Institutional Review Board (IRB) of Seoul National University Hospital (IRB No. 1904-0541027) and written consent forms were obtained from all parents or their legal representatives. All medical records were reviewed and home videos of patients with movement symptom were collected and analyzed independently by 2 pediatric neurologists.

\section{Whole exome sequencing and variant identification}

Of the 6 families, 3 underwent trio-WES and the other 3 had only the probands sequenced. Genomic DNA was extracted from peripheral blood leucocytes using a QIAamp DNA Blood Midi Kit according to the manufacturer's instructions (Qiagen, Valencia, CA, USA). WES procedures including exome capturing and sequencing were performed at Theragen Etex Bio Institute (Suwon, Korea). The sequenced reads were aligned to human reference genome patch 13 (GRCh37.p13) using a Burrows-Wheeler Aligner (version 0.7.15). Picard software (version 2.8.0), SAMtools (version 1.8), and a Genome Analysis Toolkit (GATK, version 4.1.4) were used for further data processing such as removal of polymerase chain reaction (PCR) duplicates, base recalibration, and variant quality control. All variants were called using the GATK HaplotypeCaller in GVCF mode, and the called variants were annotated using ANNOVAR and SnpEff. The pathogenicity of variants was evaluated according to the American College of Medical Genetics (ACMG) standards guidelines [14]. Segregation test was done for 3 proband-WES cases using Sanger sequencing.

\section{Amplicon sequencing}

Amplicon sequencing was conducted for 1 family (patient 5) to identify parental mosaicism (Fig. 1). The primer was designed to amplify genomic region of interest. It can create a single amplicon of approximate $200 \mathrm{bp}$ and the target position has a distance of $100 \mathrm{bp}$ or less from the $5^{\prime}$ end. Six nucleotide barcode and adaptor sequences were added to the $5^{\prime}$ end of the primers to identify family members. PCR procedures were performed as previously described in detail [15]. Next-generation sequencing was performed using a dual indexing strategy and PCR free kit by Theragen Etex Bio Institute (Suwon, Korea).

\section{Results \\ Overall clinical features}

Three female and 3 male patients were enrolled and evaluated. All clinical features are summarized in Table 1. Five patients were referred to the clinic due to hypotonia or global developmental delay which was noticed at different ages ( 3 months old on median, range 0-75 months). Four patients (patients 2-5) had severely retarded development in motor, language, and social manner. Patient 4 showed weak crying and respiratory difficulty after birth, and was treated in a neonatal intensive care unit. She started unsteady gait without support at 4 years old, but no further achievement was shown till the most recent follow-up at her age of 8.8 years. Only 1 case (patient 4) had focal epilepsy, which started at 6 years old and was well controlled with valproate monotherapy. Patient 3 had neither movement disorder nor epilepsy. He presented as having infantile hypotonia and profound developmental delay. Generalized spasticity developed and progressed over time, dominantly on lower extremities.

\section{Phenotypic spectrum of movement disorder}

Movement disorder was identified in 5 patients in a different manner. Patient 1 initially visited the rehabilitation clinic because of poor hand skills at 6 years old, but no further tests were given because her symptoms were quite subjective without any progression nor abnormalities on neurological examination. However, intermittent nonepileptic truncal myoclonus followed by focal 


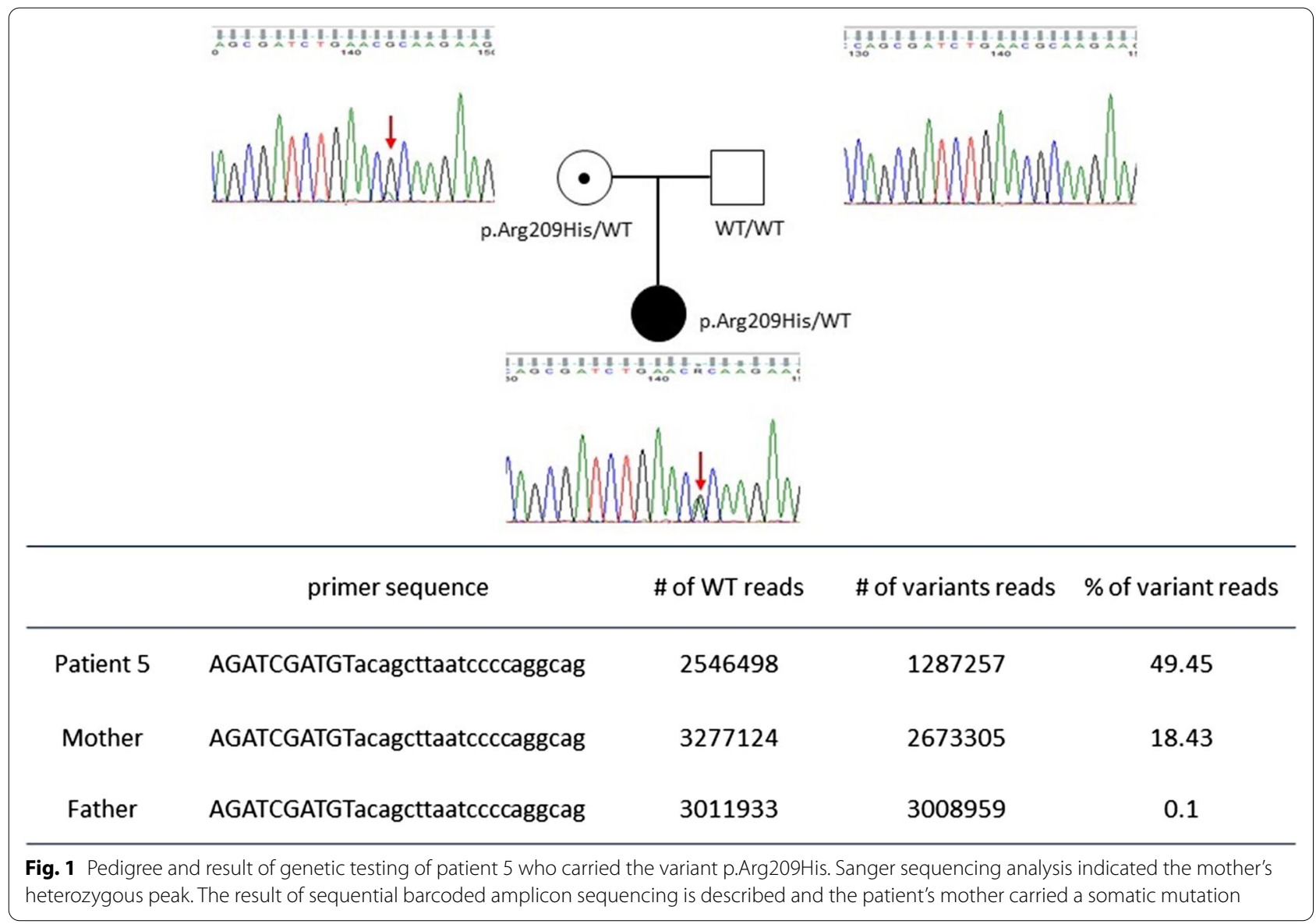

dystonic gait was recognized at the age of 10 years. She underwent several genetic tests for dystonia including Segawa disease, but no sequence variants in known genes were noticed. Her symptoms had progressed slowly and spasticity of lower extremities and increased knee jerk was noted at the latest clinical follow-up (17 years old).

Patient 2 started his choreoathetosis around 2 years old. His chorea was accompanied by brief focal dystonia, which lasted all day long and disappeared during sleep. It was not much deteriorated with advancing age (see Additional file 1).

Patient 5 was initially reported to have intermittent hyperkinesia with brief jerking and truncal dystonia triggered by emotional upset which started at 1 year old (see Additional file 2). At the age of 5 years, orofacial dyskinesia developed and became prominent (see Additional file 3). Brief dystonia on her neck and shoulder also occurred and her movement presentations progressed over time (see Additional file 4, at 8 years old). Eventually she found it difficult to walk or crawl. Her movements showed acute exacerbations during febrile illness.

Patient 6 showed ataxia before 1 year of age without significant worsening. He started walking independently at 16 months old, but his gait has remained unstable till the most recent follow-up. Brief focal dystonia of lower extremities was also noted during walking (see Additional files 5 and 6 , at 24 and 27 months old, respectively).

\section{Mutation analysis and genotype-phenotype association}

Six different variants from 6 patients were identified using WES (Table 1). Patients 1-3 underwent trio-WES whereas patients 4-6 had WES for proband only. All variants were classified as pathogenic or likely pathogenic according to the ACMG guidelines. Five variants were confirmed to be de novo mutations, but patient 5 inherited her variant from her mother who carried the mosaic variant, a state identified through amplicon sequencing (Fig. 1). In this cohort, 4 variants (from patients 2, 4, 5, and 6) were located in a mutational hot spot and 3 of them (p.Glu246Lys of patient 2, p.Ala227Val of patient 4 , p.Arg209His of patient 5) were reported previously $[2,4,6,7]$. We reviewed previously reported cases and compared detailed phenotypes (Table 2). Patients with the variant of p.Glu246Lys or p.Arg209His showed quite homogeneous phenotypes: e.g., infantile hypotonia, profound development delay, or severe choreoathetosis 
Table 1 Clinical features of six patients with GNAO1 variants

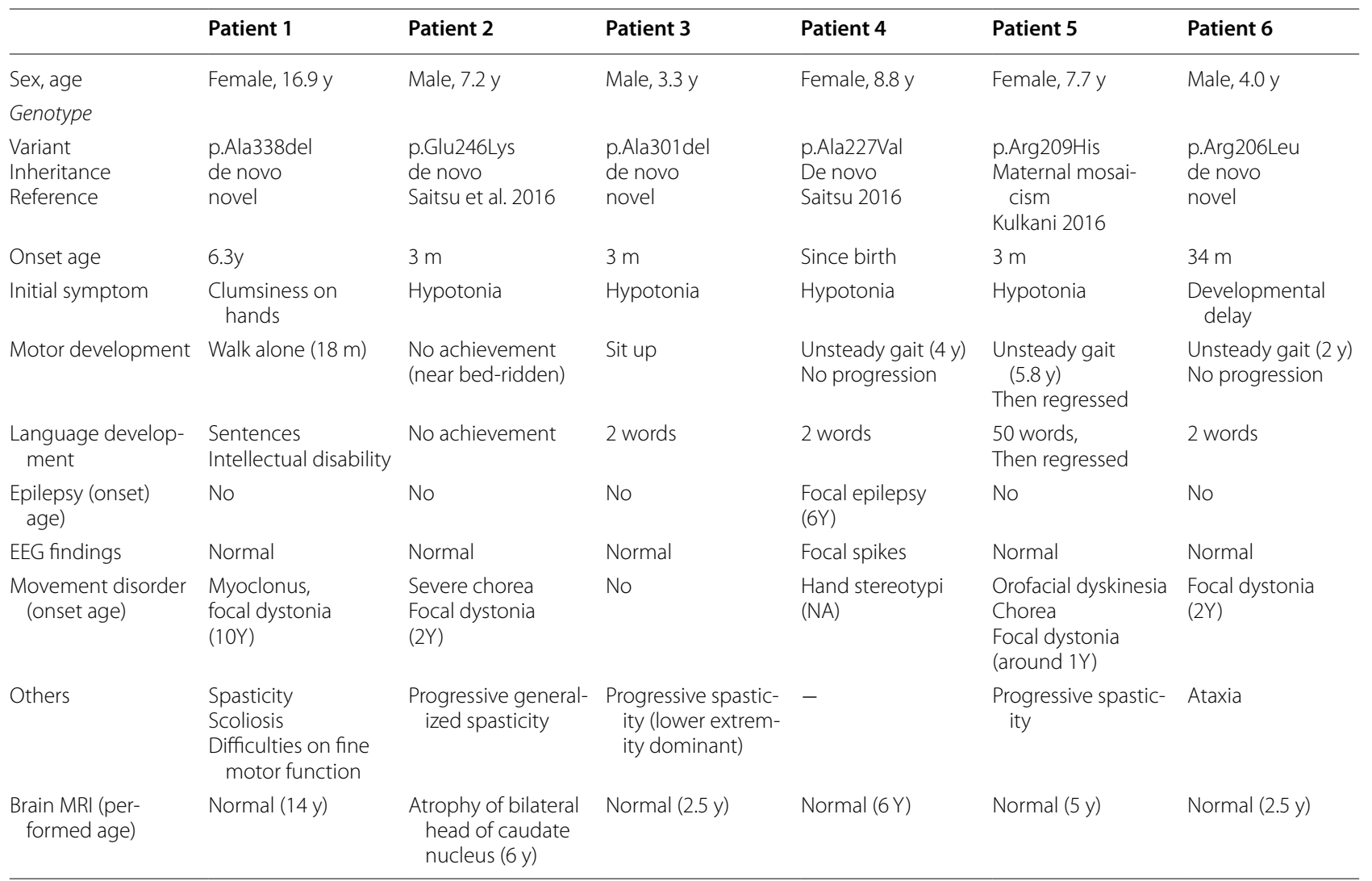

$y$, years; $m$, months

started in early childhood. In particular, patients with p.Arg209His were reported to have severe exacerbation and required multiple admissions.

\section{Discussion}

Movement disorder is difficult to evaluate, especially in children, because they are in a developmental process and their symptoms evolve over time. Comprehensive phenotyping is quite important for diagnosis of these patients, even in the genomic era. In our cohort, all except 1 patient had multiple genetic testing including diagnostic exome sequencing and gene panel sequencing before participation in KUDP. After the diagnosis, we reviewed the patient's clinical course including previous videos. Patients 1 and 3 showed atypical presentations. We also found some quite unique presentations of patients with some recurrent GNAO1 variants (p.Glu246Lys and p.Arg209His). As previously reported, patients initially presented with profound infantile hypotonia and developmental delay, and severe choreoathetosis movement developed in all patients during early childhood $[2,4,6$, 7]. Patient 2 in our cohort showed movement symptoms since he was about 2 years old. The symptoms persisted, but were not much progressed or suddenly exacerbated, as reported in patients with a p.Glu246Lys variant. Patient 5 was reported to have orofacial dyskinesia starting at 4 years old, but was eventually found to have early hyperkinetic movement. She was also admitted several times for pneumonia and accompanying aggravation of neck dystonia as in the previous cases [2,6]. This indicated a quite homogeneous and characteristic clinical course with high suspicion of GNAO1 encephalopathy. Most of those patients visited a clinic for their hypotonia or developmental delay, which are nonspecific and common for a pediatric neurologist. However, if we noticed unexplained early onset dystonia or chorea as well as severe developmental delay, GNAO1 might be initially considered as a genetic cause. Early diagnosis based on clinical presentation is important because of additional treatment options such as deep brain stimulation and tetrabenazine for severe movement phenotypes $[2,9,16]$.

It is obvious that a genotype-phenotype correlation exists, at least in certain loci. Many functional studies were performed, especially for recurrent mutations to date. Some variants work as GOM, whereas others work as loss-of-function mutations $[1,2,11]$. Different 
Table 2 Phenotype review of the patients with the variant p.Glu246Lys and p.Arg209His

\begin{tabular}{|c|c|c|c|c|c|c|c|c|}
\hline No & Reference & Age/sex & $\begin{array}{l}\text { Initial symptom } \\
\text { (onset age) }\end{array}$ & $\begin{array}{l}\text { Epilepsy (onset } \\
\text { age) }\end{array}$ & $\begin{array}{l}\text { Movement } \\
\text { disorder (onset } \\
\text { age) }\end{array}$ & $\begin{array}{l}\text { Max motor } \\
\text { achievement }\end{array}$ & $\begin{array}{l}\text { Max speech } \\
\text { achievement }\end{array}$ & Brain MRI \\
\hline \multicolumn{9}{|c|}{ p.Glu246Lys } \\
\hline 1 & $\begin{array}{l}\text { This report } \\
\text { (patient 2) }\end{array}$ & M/7.2 y & Hypotonia (3 m) & None & $\begin{array}{l}\text { Chorea, dystonia } \\
(2 \mathrm{y})\end{array}$ & None & None & $\begin{array}{l}\text { Atrophy of bilateral } \\
\text { head of caudate } \\
\text { nucleus }(6 y)\end{array}$ \\
\hline 2 & Saitsu ${ }^{7}$ & $F / 13 y$ & $\begin{array}{c}\text { Developmental } \\
\text { delay }(4 \mathrm{~m})\end{array}$ & None & $\begin{array}{l}\text { Severe athetosis } \\
\text { (NA) }\end{array}$ & None & None & Normal (12 y) \\
\hline $3^{*}$ & Ananth 6 & M/5.5 y & Hypotonia (3 m) & None & Chorea (4 y) & None & None & Normal (12 m) \\
\hline $4^{*}$ & Ananth $^{6}$ & $F / 5.5 y$ & Hypotonia (3 m) & None & Chorea (4 y) & None & None & $\begin{array}{l}\text { Global atrophy } \\
\quad(5.5 \mathrm{y})\end{array}$ \\
\hline 5 & Ananth 6 & $F / 10.3 y$ & Hypotonia (6 m) & None & Chorea (4 y) & None & None & $\begin{array}{l}\text { Global atrophy, T2 } \\
\text { hypointensity } \\
\text { in globus pallidi } \\
(9 \mathrm{y})\end{array}$ \\
\hline 6 & Ananth ${ }^{6}$ & M/15 y & Hypotonia (5 m) & None & Chorea (4 y) & None & $\begin{array}{l}\text { None (simple } \\
\text { non-verbal } \\
\text { communica- } \\
\text { tion) }\end{array}$ & $\begin{array}{l}\text { T2 hypointensity } \\
\text { in globus pallidi } \\
\text { (14y) }\end{array}$ \\
\hline $7 \dagger$ & Schorling ${ }^{4}$ & M/8 y & $\begin{array}{l}\text { Myoclonic } \\
\text { twitching (1 m) }\end{array}$ & None & $\begin{array}{l}\text { Myoclonus (1 m), } \\
\text { Dystonia }(2 \mathrm{y})\end{array}$ & None & NA & Normal (18 m) \\
\hline $8+$ & Schorling 4 & $F / 3 y$ & $\begin{array}{l}\text { Developmental } \\
\text { delay }(5 \mathrm{~m})\end{array}$ & $\begin{array}{l}\text { Focal epilepsy } \\
(7 \mathrm{~m})\end{array}$ & Dystonia (NA) & Head control & NA & $\begin{array}{l}\text { Atrophy, thin } \\
\text { corpus callosum } \\
(2 \mathrm{y})\end{array}$ \\
\hline \multicolumn{9}{|c|}{ p.Arg209His } \\
\hline 1 & $\begin{array}{l}\text { This report } \\
\text { (patient 5) }\end{array}$ & F/7.7 y & Hypotonia (3 m) & None & $\begin{array}{l}\text { Orofacial dyski- } \\
\text { nesia } \\
\text { Chorea } \\
\text { Focal dystonia } \\
\text { Myoclonus ( } 2 \text { y) }\end{array}$ & Stand (regressed) & 50 words & Normal (5 y) \\
\hline $2 \ddagger$ & Kulkani $^{2}$ & M/8 y & Hypotonia (18 m) & None & $\begin{array}{l}\text { Sever Chorea } \\
\text { Athetosis (34 m) }\end{array}$ & NA & NA & Normal (7 y) \\
\hline $3 \neq$ & Kulkani ${ }^{2}$ & M/6y & $\begin{array}{l}\text { Hyperkinesia } \\
\quad(2 \mathrm{y})\end{array}$ & None & $\begin{array}{l}\text { Severe Chorea } \\
\text { Athetosis (2 y) }\end{array}$ & NA & NA & Normal (6 y) \\
\hline 4 & Ananth 6 & M/16y & Hypomotor (6 m) & & Chorea (3 y) & Head control & $\begin{array}{l}\text { Monosyllable } \\
\text { words }\end{array}$ & $\begin{array}{l}\text { Global atrophy } \\
\text { (15y) }\end{array}$ \\
\hline
\end{tabular}

* They were dizygotic twins from non-consanguineous parents

+ They were siblings from non-consanguineous parents

₹ They were siblings from on-consanguineous pare

functional alteration may contribute to different phenotypes of GNAO1 encephalopathy [11, 12]. Further studies are expected to evaluate other variants in GNAO1 and its related pathway, which will allow us to know more about the disease and its possible treatment.

We verified maternal mosaicism from 1 family (patient 5 ) by additional amplicon sequencing. We suspected mosaicism in this patient, based on confirmative Sanger sequencing for the patient's mother, indicating a low heterozygous peak. This verification is crucial for the family counselling. Three familial cases were already reported in spite of a small number of total patients, which suggested parental mosaicism might be common in GNAO1 encephalopathy [2, 4, 6, 17].
Therefore, testing for the mosaicism should be conducted if parents have plan to have another child.

\section{Conclusions}

This study reported 6 cases of GNAO1 encephalopathy focusing on their movement phenotypes with their video clips (Additional files). Early-onset chorea with profound developmental problems is quite characteristic for patients with a movement-dominant phenotype. We also reported atypical and novel findings for GNAO1 encephalopathy including proven mosaicism in parents, which is important to guide genetic counselling. 


\section{Supplementary information}

Supplementary information accompanies this paper at https://doi. org/10.1186/s13023-020-01594-3.

Additional file 1: Chreoathetosis with multifocal brief dystonia in Patient 2 (at 4 years old).

Additional file 2: Hyperkinesia and choreoathetosis in Patient 5 (at 1 year old).

Additional file 3: Orofacial dyskinesia in Patient 5 (at 4 years old).

Additional file 4: Sustained choreoathetosis with spasticity on lower extremities and orofacial dyskinesia (at 8 years old).

Additional file 5: Focal mild dystonic gait (at 24 months old).

Additional file 6: Focal dystonic gait with mild ataxia (at 27 months old).

\section{Acknowledgements}

We greatly appreciate the contributions to DNA preparation by the pediatric neurology laboratory at Seoul National University Children's Hospital. WES sequencing were performed at Theragen Etex Co. Ltd. (Seoul, Korea).

\section{Authors' contributions}

SYK contributed to the data acquisition, prepared the first draft of the manuscript, and edited the manuscript drafts. YKS and YJK contributed to the data acquisition. SP and JSS managed all the process of WES, Sanger sequencing, and amplicon sequencing including data acquisition and analysis. BCL and KJK contributed to acquisition and analysis of patients' movement profiles with their serial video clips. JHC reviewed patients' video clips and edited the manuscript drafts until the final draft was produced and mentored SYK through the process by correspondence. All authors read and approved the final manuscript." in the "Authors' contributions" section.

\section{Funding}

This study was supported by a research program funded by the Korea Centers for Disease Control and Prevention (Grant No. 2018-ER6901-02).

\section{Availability of data and materials}

All data generated or analyzed for the study are available from the corresponding author upon reasonable request.

\section{Ethics approval and consent to participate}

The study protocol was in accordance with the tenets of Declaration of Helsinki and was approved by the Institutional Review Board of Seoul National University.

\section{Consent for publication}

Three families agreed to report the case with patients' video clips. Independent written informed consents were obtained from the parents. We greatly appreciate the patients and their families for providing their videos and agreeing to this study.

\section{Competing interests}

The authors have no conflict of interest or financial relationship to disclose.

\section{Author details}

${ }^{1}$ Division of Pediatric Neurology, Department of Pediatrics, Pediatric Clinical Neuroscience Center, Seoul National University Children's Hospital, Seoul, Korea. ${ }^{2}$ Rare Disease Center, Seoul National University Hospital, Seoul, Korea. ${ }^{3}$ Department of Medicine, Seoul National University College of Medicine Graduate School, Seoul, Korea. ${ }^{4}$ Department of Pediatrics, Seoul National University College of Medicine, Seoul, Korea.

Received: 17 August 2020 Accepted: 26 October 2020

Published online: 09 December 2020

\section{References}

1. Nakamura K, Kodera H, Akita T, Shiina M, Kato M, Hoshino H, et al. De Novo mutations in GNAO1, encoding a Galphao subunit of heterotrimeric $G$ proteins, cause epileptic encephalopathy. Am J Hum Genet. 2013;93(3):496-505.

2. Kulkarni N, Tang S, Bhardwaj R, Bernes S, Grebe TA. Progressive movement disorder in brothers carrying a GNAO1 mutation responsive to deep brain stimulation. J Child Neurol. 2016;31(2):211-4.

3. Gawlinski P, Posmyk R, Gambin T, Sielicka D, Chorazy M, Nowakowska B, et al. PEHO syndrome may represent phenotypic expansion at the severe end of the early-onset encephalopathies. Pediatr Neurol. 2016;60:83-7.

4. Schorling DC, Dietel T, Evers C, Hinderhofer K, Korinthenberg R, Ezzo D, et al. Expanding phenotype of de novo mutations in GNAO1: four new cases and review of literature. Neuropediatrics. 2017;48(5):371-7.

5. Arya R, Spaeth C, Gilbert DL, Leach JL, Holland KD. GNAO1-associated epileptic encephalopathy and movement disorders: c.607G>A variant represents a probable mutation hotspot with a distinct phenotype. Epileptic Disord. 2017;19(1):67-75.

6. Ananth AL, Robichaux-Viehoever A, Kim YM, Hanson-Kahn A, Cox R, Enns GM, et al. Clinical course of six children with GNAO1 mutations causing a severe and distinctive movement disorder. Pediatr Neurol. 2016;59:81-4.

7. Saitsu H, Fukai R, Ben-Zeev B, Sakai Y, Mimaki M, Okamoto N, et al. Phenotypic spectrum of GNAO1 variants: epileptic encephalopathy to involuntary movements with severe developmental delay. Eur J Hum Genet. 2016;24(1):129-34

8. Talvik I, Moller RS, Vaher M, Vaher U, Larsen LH, Dahl HA, et al. Clinical phenotype of de novo GNAO1 mutation: case report and review of literature. Child Neurol Open. 2015;2(2):2329048X15583717.

9. Waak M, Mohammad SS, Coman D, Sinclair K, Copeland L, Silburn P, et al. GNAO1-related movement disorder with life-threatening exacerbations: movement phenomenology and response to DBS. J Neurol Neurosurg Psychiatry. 2018;89(2):221-2.

10. Menke LA, Engelen M, Alders M, Odekerken VJ, Baas F, Cobben JM. Recurrent GNAO1 mutations associated with developmental delay and a movement disorder. J Child Neurol. 2016;31(14):1598-601.

11. Feng H, Sjogren B, Karaj B, Shaw V, Gezer A, Neubig RR. Movement disorder in GNAO1 encephalopathy associated with gain-of-function mutations. Neurology. 2017;89(8):762-70.

12. Feng $H$, Khalil $S$, Neubig RR, Sidiropoulos $C$. A mechanistic review on GNAO1-associated movement disorder. Neurobiol Dis. 2018;116:131-41.

13. Kim SY, Lim BC, Lee JS, Kim WJ, Kim H, Ko JM, et al. The Korean undiagnosed diseases program: lessons from a one-year pilot project. Orphanet J Rare Dis. 2019;14(1):68.

14. Richards S, Aziz N, Bale S, Bick D, Das S, Gastier-Foster J, et al. Standards and guidelines for the interpretation of sequence variants: a joint consensus recommendation of the American College of Medical Genetics and Genomics and the Association for Molecular Pathology. Genet Med. 2015;17(5):405-24.

15. Kim S, Han J, Kim HA, Lim BC, Seo JE, Choi M, et al. Neuropathy, ataxia, retinitis pigmentosa-like phenotype associated with a mitochondrial G8363A mutation in a family. Ann Clin Lab Sci. 2018;48(4):546-8.

16. Danti FR, Galosi S, Romani M, Montomoli M, Carss KJ, Raymond FL, et al. GNAO1 encephalopathy: broadening the phenotype and evaluating treatment and outcome. Neurol Genet. 2017;3(2):e143.

17. Monies D, Abouelhoda M, Assoum M, Moghrabi N, Rafiullah R, Almontashiri N, et al. Lessons learned from large-scale, first-tier clinical exome sequencing in a highly consanguineous population. Am J Hum Genet. 2019;104:1182-201.

\section{Publisher's Note}

Springer Nature remains neutral with regard to jurisdictional claims in published maps and institutional affiliations. 